\title{
Undulatory swimming in viscoelastic fluids
}

Phys. Rev. Lett. 106 (2011) 208101

Xiaoning Shen and P. E. Arratia

Recommended with a commentary by Thomas R. Powers, Brown University

It is a common observation that many microorganisms swim in water. At micron scales, water is very viscous, inertia is unimportant, and the fluid mechanics is described by low Reynolds number hydrodynamics [1]. The fundamental theory of swimming at low Reynolds number in a Newtonian liquid such as water is well-developed, although the full consequences of this theory are still being worked out [1]. On the other hand, many swimmers encounter fluids that have an elastic as well as a viscous response. For example, microrganisms ranging from infectious bacteria to mammalian sperm swim in mucus, a gel of cross-linked polymers pervaded by water. The fundamental principles of swimming in non-Newtonian fluids such as gels and polymer solutions are only beginning to emerge.

How do the properties of the fluid affect the speed of a swimmer? Consider the prescribed swimmer problem, where the body of the swimmer undergoes prescribed deformations independent of the properties of the medium. In 1951, G. I. Taylor calculated the swimming speed for an infinite sheet with low-amplitude propagating bending waves in a highly viscous, Newtonian fluid [2]. He found that the swimming speed is $U=c b^{2} q^{2} / 2$, where $c$ is the speed of the propagating wave, $b$ is the wave amplitude, and $q$ is the wavenumber. He also found a similar expression for an infinite cylinder with low-amplitude propagating bending waves [3]. In both cases, the swimming speed is independent of viscosity, since the parameters of the motion $-c, q$, and $b$-are prescribed independent of the load.

Adding polymers to water yields a medium with elastic as well as viscous properties. The elasticity is characterized by the relaxation time, the time it takes for a polymer stretched out by shear flow to relax to its equilibrium configuration once the flow ceases. For sperm or bacteria in mucus, the relaxation time can be comparable to or even larger than the characteristic beat frequency, indicating that elasticity is important. For a dilute polymer solution, Lauga analytically calculated that Taylor's swimming sheet moves more slowly than the Newtonian case, with speed ${ }^{1} U=c b^{2} q^{2} /\left(1+\mathrm{De}^{2}\right) / 2$, where the Deborah number De is the ratio of the relaxation time and the beat period [4]. Fu et al. found a similar formula for waves on an infinite filament [5]. Note that the relaxation time, a material property, appears in the swimming speed for case of a polymer solution, even though the swimming motion is prescribed. Teran et al. used numerical techniques to calculate the swimming speed of a finite-length swimmer undergoing large-amplitude deformations, with the amplitude increasing with distance from the head of the swimmer as in a mammalian sperm [6]. They found in this case that the swimming speed first increases with Deborah number, peaking around unity, and then eventually decreases below the Newtonian value.

Recently, Shen and Arratia have made the first quantitative measurements of the swimming speed of a biological microswimmer in a polymer solution [7]. They use the nematode Caenorhabditis elegans, a worm about $1 \mathrm{~mm}$ long and $80 \mu \mathrm{m}$ in diameter that

\footnotetext{
${ }^{1}$ Here we only discuss the case of solution viscosity much less than the polymer viscosity.
} 
normally swims in soil saturated with water. Shen and Arratia used the worm as a model swimmer, suspending it in a dilute solution of carboxymethyl cellulose (CMC) in water. The relaxation time of the solution is varied by roughly a factor of ten by varying the concentration of CMC. The worm is not exactly a prescribed swimmer: the frequency of its beat pattern decreases with viscosity, which depends on the CMC concentration. However, the wavenumber and amplitude stay fixed. Thus, Shen and Arratia plot the swimming speed versus Deborah number, and find that speed decreases with Deborah number. Normalizing the swimming speed measured in the viscoelastic fluid by the swimming speed measured in a Newtonian fluid of the same viscosity, they find rough agreement with the predicted swimming speed of references [4] and [5], normalized by the experimentally measured speed in the Newtonian fluid. Exact agreement is not expected, since the worm has finite length and has large-amplitude waves that are not purely sinusoidal since the amplitude decreases with distance from the head. Shen and Arratia also measure the fluid flow around the swimming worm. Both the Newtonian and non-Newtonian flow fields exhibit hyperbolic points; the authors suggest that the enhanced stress near these points in the viscoelastic fluid leads to the reduction in swimming speed relative to the Newtonian fluid. These experiments represent a substantial advance in our understanding of how microorganisms swim in viscoelastic fluids. The next experimental challenge is to make similar measurements on even smaller microorganisms, as has recently been done for algae in Newtonian fluids $[8,9]$.

\section{References}

[1] E. Lauga and T. R. Powers. The hydrodynamics of swimming microorganisms. Rep. Prog. Phys., 72:096601, 2009.

[2] G. I. Taylor. Analysis of the swimming of microscopic organisms. Proc. R. Soc. Lond. Ser. A, 209:447-461, 1951.

[3] G. I. Taylor. The action of waving cylindrical tails in propelling microscopic organisms. Proc. R. Soc. Lond. A Phys. Sci., 211:225, 1952.

[4] E. Lauga. Propulsion in a viscoelastic fluid. Phys. Fluids, 19:083104, 2007.

[5] H. C. Fu, T. R. Powers, and C. W. Wolgemuth. Theory of swimming filaments in viscoelastic media. Phys. Rev. Lett., 99:258101-258105, 2007.

[6] J. Teran, L. Fauci, and M. Shelley. Viscoelastic fluid response can increase the speed and efficiency of a free swimmer. Phys. Rev. Lett., 104:038101, 2010.

[7] X. N. Shen and P. E. Arratia. Undulatory swimming in viscoelastic fluids. Phys. Rev. Lett., 106:208101, 2011.

[8] K. Drescher, R. Goldstein, N. Michel, M. Polin, and I. Tuval. Direct Measurement of the Flow Field around Swimming Microorganisms. Phys. Rev. Lett., 105:168101, 2010.

[9] J. Guasto, K. Johnson, and J Gollub. Oscillatory Flows Induced by Microorganisms Swimming in Two Dimensions. Phys. Rev. Lett., 105:168102, 2010. 